\title{
Maximal functions and Hilbert transforms associated to polynomials
}

\section{Anthony Carbery, Fulvio Ricci and James Wright}

\section{Introduction.}

Let $M$ denote the classical Hardy-Littlewood maximal function

$$
M f(x)=\sup _{h>0} \frac{1}{2 h} \int_{-h}^{h}|f(x-t)| d t
$$

and $H$ the classical Hilbert transform

$$
H f(x)=\text { p.v. } \int_{-\infty}^{\infty} f(x-t) \frac{d t}{t},
$$

on $\mathbb{R}^{1}$. The mapping properties of these functions are very well-known (see for example [S1]), as are those of their higher dimensional analogues the Hardy-Littlewood-Wiener maximal function and the Calderón-Zygmund singular integral operators. Analogues of $M$ and $H$ associated to certain submanifolds of positive codimension in $\mathbb{R}^{n}, n \geq 2$, have also been extensively studied. These are the so-called maximal functions and singular integrals along surfaces, or maximal and singular Radon transforms. See for example [SW], [S2], [Ch], [PS1], [RS2], [CWW1], [CWW2]. One approach to these general problems is to model them on translation-invariant problems in certain homogeneous Lie groups so that the basic translation operation $(x, t) \longmapsto x-t$ on $\mathbb{R}^{n} \times \mathbb{R}^{n}$ is 
replaced by $(x, t) \longmapsto x t^{-1}$ on the Lie group. When written in terms of canonical coordinates, this multiplication is a polynomial mapping. Another approach, at least for the singular integral problems, is via oscillatory integrals and Fourier integral operators. In certain model cases a partial Fourier transform may be used to reduce the problem to a less singular one but with the familiar difference or inner product replaced by a more general mapping on $\mathbb{R}^{n} \times \mathbb{R}^{n}$. Once again, polynomial mappings provide substantial model cases in this setting. Thus an understanding of the classical operators of harmonic analysis with translation and inner product replaced by more general polynomial mappings is an important step in the study of higher dimensional problems associated to submanifolds.

However, very little seems to have been done systematically in this direction, with the principal exception of [RS1], [RS2], [PS2] and [HP]. In the present paper we take up this point in the context of the most classical one-dimensional operators of harmonic analysis, the HardyLittlewood maximal function and the Hilbert transform. While we do not believe our results will have any direct bearing on the higher dimensional problems mentioned above, it nevertheless seems a reasonable starting point to consider the one-dimensional setting first.

Thus we let $\mathfrak{p}: \mathbb{R} \times \mathbb{R} \longrightarrow \mathbb{R}$ be a polynomial mapping $\mathfrak{p}:(x, t) \longmapsto$ $\mathfrak{p}(x, t)$. We shall assume that $\mathfrak{p}$ has degree $n \geq 1$ in the second variable and that $\mathfrak{p}(x, 0)=x$. (That this condition cannot be entirely dispensed with is discussed below, and is natural in so far as the averages occurring in $M_{\mathfrak{p}}$ below are then concerned with the local behaviour of $f$ near $x$.) We define the maximal function and Hilbert transform associated to $\mathfrak{p}$ as

$$
M_{\mathfrak{p}} f(x)=\sup _{h>0} \frac{1}{2 h} \int_{-h}^{h}|f(\mathfrak{p}(x, t))| d t
$$

and

$$
H_{\mathfrak{p}} f(x)=\mathrm{p} \cdot \mathrm{v} \cdot \int_{-\infty}^{\infty} f(\mathfrak{p}(x, t)) \frac{d t}{t}
$$

when these make sense. (Indeed, as a consequence of Theorem 2.4 below, $H_{\mathfrak{p}}$ can be realised as a principal-value distribution.) When $\mathfrak{p}(x, t)=x-p(t)$ - with $p$ a polynomial of degree $n$ of one real variable $t$ satisfying $p(0)=0-$ we sometimes write these as $M_{p}$ and $H_{p}$. The main object of this paper is to begin to study the mapping properties of these operators.

The principal results are as follows: 
Theorem 1. If $\mathfrak{p}$ has degree $n$ in $t$, then $M_{\mathfrak{p}}$ and $H_{\mathfrak{p}}$ are bounded on $L^{p}(\mathbb{R})$ when $p>n$. $M_{\mathfrak{p}}$ and $H_{\mathfrak{p}}$ may not be bounded on $L^{n}(\mathbb{R})$ for certain $\mathfrak{p}$ of degree $n$ in $t$.

Theorem 2. If $\mathfrak{p}$ is quadratic in $t$, then the mapping properties of $M_{\mathfrak{p}}$ and $H_{\mathfrak{p}}$ can be precisely given terms of the behaviour of the coefficients of $t$ and $t^{2}$ in $\mathfrak{p}$. (See Theorem 3.2 for full details.)

Theorem 3. If $\mathfrak{p}(x, t)=x-p(t)$, then the operators $M_{p}$ and $H_{p}$ are of weak-type 1-1 with bounds depending only on the degree $n$ of $p$, and not otherwise on the coefficients.

These theorems are proved in subsections 3.1, 3.2 and 3.3 respectively of Section 3 .

As the conditions of Theorem 1.1 place no constraints on the (polynomial) coefficients of $t$ whatsoever, it is natural to consider the situation when these coefficients of $t$ are completely arbitrary functions of $x$. Thus we are lead to what we term the supermaximal function and superhilbert transform, which seem to be of independent interest. These are defined as

$$
\mathcal{M}_{n} f(x)=\sup _{p \in \mathfrak{P}_{n}} M_{p} f(x)=\sup _{\substack{h>0 \\ p \in \mathfrak{P}_{n}}} \frac{1}{2 h} \int_{-h}^{h}|f(x-p(t))| d t
$$

and

$$
T_{n} f(x)=\sup _{p \in \mathfrak{P}_{n}}\left|H_{p} f(x)\right|=\sup _{p \in \mathfrak{P}_{n}}\left|\int_{-\infty}^{\infty} f(x-p(t)) \frac{d t}{t}\right|,
$$

where $\mathfrak{P}_{n}$ is the class of polynomials $p$ of degree at most $n$ in $t$ with $p(0)=0$. The result about these operators, proved in Section 2, is the following:

Theorem 1.4. $\mathcal{M}_{n}$ and $T_{n}$ are bounded on $L^{q}(\mathbb{R})$ if and only if $q>n$.

An interesting lemma that we use to prove these results is that $\left|H_{p} f(x)\right|$ is pointwise dominated by $M_{p} f(x)$ plus the maximal Hilbert transform $H^{*} f(x)$ with constants depending only on the degree of $p$. $\left(H^{*} f(x)\right.$ is defined as

$$
\sup _{0<a<b<\infty}\left|\int_{a<|t|<b} f(x-t) \frac{d t}{t}\right|,
$$


and it is well-known (see for example [S1]) that this operator is of weaktype 1-1.)

We comment upon the condition $\mathfrak{p}(x, 0)=x$. This comes from the analogues in higher dimensions where one wants to think geometrically of $S(x, t)$ as, for each fixed $x$, some surface passing through $x$ when $t=0$. However there is no particular reason to assume $\mathfrak{p}(x, 0)=x$ in our setting other than that a necessary condition for any $L^{p}(p<\infty)$ boundedness of $M_{\mathfrak{p}}$ is that $\mathfrak{p}(x, 0)$ have no critical points. (To see this, suppose $\mathfrak{p}(x, 0)$ has a critical point at say zero. Then for $\delta$ sufficiently small, $|x| \leq C \delta^{1 / 2}$ and $|t| \leq C \delta$ implies $|\mathfrak{p}(x, t)| \leq C^{\prime} \delta$. Thus for $f=\chi_{(-\delta, \delta)}$,

$$
\frac{1}{2 h} \int_{-h}^{h} f(\mathfrak{p}(x, t)) d t \geq 1, \quad \text { if }|x| \leq C \delta^{1 / 2} \text { and } h \leq C \delta .
$$

Hence $\left\|M_{\mathfrak{p}} f\right\|_{p} \geq C \delta^{1 /(2 p)}$ while $\|f\|_{p} \sim \delta^{1 / p}$. This is a contradiction unless $p=\infty)$. If $\mathfrak{p}(x, 0)$ does have no critical points, then one can in principle change variables to reduce to the case $\mathfrak{p}(x, 0)=x$, but for modified maximal functions and Hilbert transforms whose coefficients are no longer polynomials. It is partly for this reason that we have stated Theorem 3.2 below for coefficients which are not necessarily polynomials.

Finally we make some remarks about possible higher-dimensional analogues of our results. We first note that there is no interesting supermaximal function or superhilbert transform, even of degree 1 , in $\mathbb{R}^{d}$, $d \geq 2$. This is because the putative supermaximal function contains the universal maximal function associated to averages in arbitrary directions in $\mathbb{R}^{d}$, which is well known to be unbounded on all $L^{p}, p<\infty$, by the Perron tree example. (See $[\mathrm{deG}]$ for example.) On the other hand one can study operators such as

$$
f \longmapsto \sup _{\substack{a, b \\ h>0}} \frac{1}{h}\left|\int_{0}^{h} f\left(x-\left(a t, b t^{2}\right)\right) d t\right|
$$

on $\mathbb{R}^{2}$ and indeed Marletta and Ricci $[\mathrm{MR}]$ have done so. Note that these operators arise in connection with Stein's and Bourgain's circular maximal function. Secondly, while it may well be true that there is an analogue of our Theorem 1.3 above in higher dimensions (indeed the $L^{p}, 1<p<\infty$, variant is true in all dimensions) there is at present a serious obstacle to proving it, which is the fact that the weak-type 1-1 
of the Hilbert transform and maximal function along a parabola in $\mathbb{R}^{2}$ are unknown. That is, while the operators

$$
f \longmapsto \sup _{h>0} \frac{1}{h}\left|\int_{0}^{h} f\left(x-\left(t, t^{2}\right)\right) d t\right|
$$

and

$$
f \longmapsto \int_{-\infty}^{\infty} f\left(x-\left(t, t^{2}\right)\right) \frac{d t}{t}
$$

are known to be bounded on $L^{p}\left(\mathbb{R}^{2}\right), 1<p<\infty$, it is not known whether they are of weak-type 1-1. See [SW]. However if $p: \mathbb{R}^{n} \longrightarrow \mathbb{R}^{n}$ is a polynomial which satisfies certain nondegeneracy conditions at 0 and $\infty$, then the higher-dimensional versions of $M_{p}$ and $H_{p}$ are of weaktype 1-1; moreover the same is true if we replace the additive structure of $\mathbb{R}^{n}$ by the group structure in any homogeneous Lie group. We plan to return to this matter in a forthcoming paper.

\section{The supermaximal function and the superhilbert trans- form.}

Let $\mathfrak{P}_{n}$ be the class of all real polynomials $p$, of a single real variable, of degree at most $n \geq 1$, such that $p(0)=0$. Define

$$
\mathcal{M}_{n} f(x)=\sup _{\substack{h>0 \\ p \in \mathfrak{P}_{n}}} \frac{1}{2 h} \int_{-h}^{h}|f(x-p(t))| d t=\sup _{p \in \mathfrak{P}_{n}} M_{p} f(x)
$$

(the "supermaximal" function of degree $n$.)

Theorem 2.1. Let $1<q<\infty$. Then $\mathcal{M}_{n}$ is bounded on $L^{q}(\mathbb{R})$ if and only if $q>n$. Moreover $\mathcal{M}_{n}$ is of restricted weak-type $n-n$.

Remark. When $n=1, \mathcal{M}_{1}$ is the classical Hardy-Littlewood maximal operator in one variable, and so there is nothing to prove in this case. We shall appeal to the result for $\mathcal{M}_{1}$ in the cases of higher $n$.

The failure of boundedness when $q \leq n$ may be seen as follows. Let $\lambda>0$ be large and let $p_{\lambda}(t)=\lambda\left(1-(1-t)^{n}\right)$. Let $f_{\beta}(t)=$ 
$|t|^{-1 / n}|\log | t||^{-\beta} \chi_{[0,1]}$. Then $f_{\beta} \in L^{n}$ if $\beta>1 / n$. Now for $x \gg 1$ we take $\lambda=x$ and $h=1$ and observe that

$$
\begin{aligned}
\int_{0}^{1} f_{\beta}\left(x-p_{\lambda}(t)\right) d t & =\int_{0}^{1} f_{\beta}\left(x(1-t)^{n}\right) d t \\
& =\int_{0}^{1} f_{\beta}\left(x t^{n}\right) d t \\
& =\frac{1}{x^{1 / n}} \int_{0}^{x^{1 / n}} f_{\beta}\left(s^{n}\right) d s \quad(\text { if } x \gg 1) \\
& =\frac{1}{x^{1 / n}} \int_{0}^{1} s^{-1}\left(\log \left|s^{n}\right|\right)^{-\beta} d s \\
& =\infty, \quad \text { if } \beta \leq 1 .
\end{aligned}
$$

Furthermore, for each $r>1$ we can find a $\beta \leq 1$ such that $f_{\beta} \in L^{n, r}$. Indeed, $f_{\beta} \in L^{n, r}$ if and only if $\beta>1 / r$. Thus $\mathcal{M}_{n}$ does not map $L^{n, r}$ to any Lebesgue-Lorentz space for any $r>1$. (See [StW] for a discussion of Lorentz spaces and related topics.)

Proof of TheOrem 2.1. We only need consider the restricted weaktype $n-n$ result as the case $q>n$ follows by interpolation with the trivial $L^{\infty}$ result, and the negative result has been established in the discussion above.

Let $S \subseteq \mathbb{R}$ be a measurable set, and let $f=\chi_{S}$. It suffices to prove that $\left\|\mathcal{M}_{n} f\right\|_{n, \infty} \leq C_{n}\|f\|_{n}$, by standard arguments from Lorentz spaces. Let $p \in \mathfrak{P}_{n}$ and $h>0$ and consider

$$
\frac{1}{h} \int_{-h}^{h} f(x-p(t)) d t=\int_{I_{h}} f(x-u) g(u) d u,
$$

where $I_{h}=p([-h, h])$,

$$
g(u)=\frac{1}{h} \sum_{j} \chi_{E_{j}}(u) \frac{1}{\left|p^{\prime}\left(p_{j}^{-1}(u)\right)\right|},
$$

where $\left\{E_{j}\right\}$ are the images under $p$ of the intervals upon which $p$ is 
monotonic, and where $p_{j}^{-1}$ is the inverse to $p$ on $E_{j}$. Then

$$
\begin{aligned}
\int_{I_{h}} f(x-u) & g(u) d u \\
& \leq\|f(x-\cdot)\|_{L^{n, 1}\left(I_{h}\right)}\|g\|_{L^{n^{\prime}, \infty}\left(I_{h}\right)} \\
& =\|f(x-\cdot)\|_{L^{n}\left(I_{h}\right)}\|g\|_{L^{n^{\prime}, \infty}\left(I_{h}\right)} \quad\left(\text { since } f=\chi_{S}\right) \\
& \leq \sup _{h>0}\left(\frac{1}{\left|I_{h}\right|} \int_{I_{h}}|f(x-u)|^{n} d u\right)^{1 / n}\left|I_{h}\right|^{1 / n}\|g\|_{L^{n^{\prime}, \infty}\left(I_{h}\right)}
\end{aligned}
$$

Now $p(0)=0$, so $0 \in I_{h}$, and thus

$$
\left(\frac{1}{\left|I_{h}\right|} \int_{\left|I_{h}\right|}|f(x-u)|^{n} d u\right)^{1 / n}
$$

is dominated by $\left(M f^{n}\right)^{1 / n}(x)$ where $M=M_{1}$ is the Hardy-Littlewood maximal function. Since

$$
\mid\left\{x:\left(M f^{n}\right)^{1 / n}(x)>\alpha|=|\left\{x: M f^{n}(x)>\alpha^{n}\right\} \mid \leq \frac{2}{\alpha^{n}} \int f^{n},\right.
$$

the result now follows once we have established the following lemma.

Lemma 2.2. There is an absolute constant $C_{n}$, depending only upon $n$, such that for all $h>0$, all $p \in \mathfrak{P}_{n}$,

$$
\left|I_{h}\right|^{1 / n}\|g\|_{L^{n^{\prime}, \infty}\left(I_{h}\right)} \leq C_{n},
$$

(Here $g$ is defined as in (1).)

Proof. For $\lambda>0$ fixed,

$$
\begin{aligned}
\left|\left\{u \in I_{h}:|g(u)|>\lambda\right\}\right| & =\int_{I_{h}} \chi_{\{u: g(u)>\lambda\}} d u \\
& =\int_{I_{h}} \chi_{\left\{u: \sum_{j} \chi_{E_{j}}(u)\left|p^{\prime}\left(p_{j}^{-1}(u)\right)\right|^{-1}>\lambda h\right\}} d u \\
& =\int_{-h}^{h} \chi_{\left\{t: 1 /\left|p^{\prime}(t)\right|>\lambda h\right\}}\left|p^{\prime}(t)\right| d t \\
& \leq \frac{1}{\lambda h}\left|\left\{t \in[-h, h]:\left|p^{\prime}(t)\right| \leq \frac{1}{\lambda h}\right\}\right| .
\end{aligned}
$$


On the other hand,

$$
\left|I_{h}\right|=\int \chi_{I_{h}}(u) d u=\int_{-h}^{h}\left|p^{\prime}(t)\right| d t
$$

Thus, to establish the lemma, it is enough to show

$$
\left|\left\{t \in[-h, h]:\left|p^{\prime}(t)\right| \leq \frac{1}{\lambda h}\right\}\right| \leq \frac{C_{n} \lambda h}{\lambda^{n /(n-1)}\left(\int_{-h}^{h}\left|p^{\prime}(t)\right| d t\right)^{1 /(n-1)}} .
$$

By scaling we may assume that $h=1$ and that $\int_{-1}^{1}\left|p^{\prime}\right|=1$ and so we are reduced to showing

$$
\left|\left\{t \in[-1,1]:\left|p^{\prime}(t)\right| \leq \alpha\right\}\right| \leq C_{n} \alpha^{1 /(n-1)}
$$

under the normalization condition $\int_{-1}^{1}\left|p^{\prime}\right|=1$.

Consider the functional $\||\cdot|\|$ on the class $Q_{n-1}$ of polynomials of degree at most $n-1$ given by

$$
\||q|\|=\max _{0 \leq j \leq n-1} \inf _{-1 \leq t \leq 1}\left|q^{(j)}(t)\right|
$$

This is a continuous function of $q$, positively homogeneous of degree 1 , which does not vanish on the unit sphere of $Q_{n-1}$, (measured, say, with respect to the $L^{1}$ norm on $\left.[-1,1]\right)$. For if $q(t)=a_{0}+a_{1} t+\cdots+a_{n-1} t^{n-1}$ and $\||q|\|=0$, we have successively that $a_{n-1}, a_{n-2}, \ldots, a_{0}$ are all zero. Thus there is a constant $m_{n}$ depending only upon $n$ such that

$$
\||q|\| \geq m_{n} \int_{-1}^{1}|q(t)| d t
$$

Applying this to $p^{\prime}$, we see that for some $j, 0 \leq j \leq n-1,\left|\left(p^{\prime}\right)^{(j)}(t)\right| \geq$ $m_{n}$ for all $t \in[-1,1]$. The mean-value theorem now yields (2) for small $\alpha$.

REMARK. (2) is an endpoint version of a result of Ricci and Stein [RS1] which states that a polynomial of degree $n-1$ (in this case $p^{\prime}$ ) is in the Muckenhoupt $A_{q}$ class, $q>n$, with constants independent of the coefficients. Inequalities such as (2) and variants in higher dimensions are also studied in [CCW]. 
We now turn to the superhilbert transform of degree $n$. Let

$$
T_{n} f(x)=\sup _{p \in \mathfrak{P}_{n}}\left|\int_{-\infty}^{\infty} f(x-p(t)) \frac{d t}{t}\right|=\sup _{p \in \mathfrak{P}_{n}}\left|H_{p} f(x)\right| .
$$

Theorem 2.3. Let $1<q<\infty$. Then $T_{n}$ is bounded on $L^{q}(\mathbb{R})$ if and only if $q>n$. Moreover $T_{n}$ is of restricted weak-type $n-n$.

Remark. Again, when $n=1, T_{1}$ is the classical Hilbert transform and so there is nothing to prove.

The negative result can be seen in a similar manner to the corresponding result for $\mathcal{M}_{n}$. Indeed, with the same $f_{\beta}$ as above, the nonintegrable singularity of $f_{\beta}$ when $\beta \leq 1$ guarantees that

$$
\int_{-\infty}^{\infty} f_{\beta}\left(x+p_{\lambda}(t)\right) \frac{d t}{t}
$$

will be $+\infty$ when $\lambda$ is taken to be $x$, at least for large $x$.

The positive part of Theorem 2.3 follows from the following result, which is also useful in other contexts.

Theorem 2.4. Let $p \in \mathfrak{P}_{n}$. Then there is the pointwise estimate

$$
\left|H_{p} f(x)\right| \leq A_{n} M_{p} f(x)+B_{n} H^{*} f(x),
$$

where $H^{*}$ is the maximal Hilbert transform and $A_{n}$ and $B_{n}$ are constants depending only upon $n$.

Proof. Let $p \in \mathfrak{p}_{n}$, and assume without loss of generality that $p$ has degree $n$ and has leading coefficient 1 . We also assume (although this is not strictly speaking necessary) that all the complex roots of $p$ are distinct. Let $0=t_{1}, t_{2}, \ldots, t_{n}$ be the $n$ complex roots of $p$ ordered so that

$$
0<\left|t_{2}\right| \leq\left|t_{3}\right| \leq \cdots \leq\left|t_{n}\right|
$$

The second and third parts of the next lemma say that the zeros of $p^{\prime}$ are strongly attracted to the zeros of $p$.

Lemma 2.5 There are constants $C(n) \geq 1$ and $\varepsilon_{0}(n)$ depending only on $n$, such that if $A>C(n)$ and $j$ and $\ell$ are such that $\ell-j \geq 3$ and are such that for some $k \in\{1, \ldots, n-1\}$

$$
\left|t_{k}\right|<A^{j}<A^{\ell}<\left|t_{k+1}\right|,
$$


then

a) If $A^{j+1} \leq|t| \leq A^{\ell-1}$,

$$
\begin{aligned}
\left(1-\frac{1}{A}\right)^{n-1}|t|^{k}\left|t_{k+1}\right| \cdots\left|t_{n}\right| & \leq|p(t)| \\
& \leq\left(1+\frac{1}{A}\right)^{n-1}|t|^{k}\left|t_{k+1}\right| \cdots\left|t_{n}\right|
\end{aligned}
$$

b) $\left|t p^{\prime}(t) / p(t)\right| \geq \varepsilon_{0}(n)$ whenever $A^{j+1} \leq|t| \leq A^{\ell-1}$,

c) $|p(t)|$ is strictly increasing on $\left[A^{j+1}, A^{\ell-1}\right]$ and strictly decreasing on $\left[-A^{\ell-1},-A^{j+1}\right]$.

Proof. a) This part is trivial since $p(t)=\prod_{m=1}^{n}\left(t-t_{m}\right)$ and, when $A^{j+1} \leq|t| \leq A^{\ell-1}$,

$$
\left(1-\frac{1}{A}\right)|t| \leq\left|t-t_{m}\right| \leq\left(1+\frac{1}{A}\right)|t|, \quad \text { for } 2 \leq m \leq k,
$$

while

$$
\left(1-\frac{1}{A}\right)\left|t_{m}\right| \leq\left|t-t_{m}\right| \leq\left(1+\frac{1}{A}\right)\left|t_{m}\right|, \quad \text { for } k+1 \leq m \leq n .
$$

(Note that only $A>1$ is required here.)

b) Observe first that

$$
\frac{p^{\prime}(t)}{p(t)}=\sum_{m=1}^{n} \frac{1}{t-t_{m}}
$$

so

$\left|\frac{p^{\prime}(t)}{p(t)}\right| \geq\left|\sum_{m=1}^{k} \frac{1}{t-t_{m}}\right|-\sum_{m=k+1}^{n} \frac{1}{\left|t-t_{m}\right|} \geq\left|\sum_{m=1}^{k} \frac{1}{t-t_{m}}\right|-\frac{(n-k)}{(A-1)|t|}$

since $\left|t_{m}\right| \geq A|t|$ if $m \geq k+1$ and $|t| \in\left[A^{j+1}, A^{\ell-1}\right]$.

Assume for simplicity that $t>0$ and consider, for $m \leq k$

$$
\operatorname{Re} \frac{1}{t-t_{m}}=\frac{t-\operatorname{Re} t_{m}}{\left|t-t_{m}\right|^{2}}>\frac{\left(1-\frac{1}{A}\right) t}{\left(1+\frac{1}{A}\right)^{2} t^{2}}=\frac{\left(1-\frac{1}{A}\right)}{\left(1+\frac{1}{A}\right)^{2}} \frac{1}{t}
$$


since $t>A\left|t_{m}\right|$.

Therefore

$$
\left|\frac{p^{\prime}(t)}{p(t)}\right| \geq\left(k \frac{\left(1-\frac{1}{A}\right)}{\left(1+\frac{1}{A}\right)^{2}}-\frac{n-k}{A-1}\right) \frac{1}{t} .
$$

Now if $A$ is sufficiently large, the coefficient of $1 / t$ is positive, which implies that $\left|t p^{\prime}(t) / p(t)\right|$ is bounded below by an absolute constant.

c) We have in fact shown that

$$
\frac{p^{\prime}(t)}{p(t)}=\operatorname{Re} \frac{p^{\prime}(t)}{p(t)}>0, \quad \text { for } t>0
$$

that is, $\log |p(t)|$ is increasing on $\left[A^{j+1}, A^{\ell-1}\right]$. Thus, $|p(t)|$ is strictly increasing on $\left[A^{j+1}, A^{\ell-1}\right]$ and similarly is strictly decreasing on $\left[-A^{\ell-1},-A^{j+1}\right]$.

In particular, if $A^{j} \leq\left|t_{2}\right| \leq A^{j+1}$, then $\left|t p^{\prime}(t) / p(t)\right|$ is bounded below and $p$ is monotonic on $\left[-A^{j-1}, A^{j-1}\right]$. One simply has to observe that, since 0 is a simple root, $p$ is monotonic through 0 .

Furthermore, implicit in the proof of Lemma 2.5 is that if $\left|t_{n}\right|<A^{j_{*}}$ and $|t| \geq A^{j_{*}+1}$ then

$$
\left(1-\frac{1}{A}\right)^{n-1}|t|^{n} \leq|p(t)| \leq\left(1+\frac{1}{A}\right)^{n-1}|t|^{n}
$$

and $\left|t p^{\prime}(t) / p(t)\right|$ is bounded below, and $|p(t)|$ is strictly increasing on $\left[A^{j_{*}+1}, \infty\right)$ and strictly decreasing on $\left(-\infty,-A^{j_{*}+1}\right]$.

A maximal set of the form $\left[-A^{\ell-1},-A^{j+1}\right] \cup\left[A^{j+1}, A^{\ell-1}\right]$ with $\ell-j \geq 3$ and such that for some $k \in\{2, \ldots, n-1\}$,

$$
\left|t_{k}\right|<A^{j}<A^{\ell}<\left|t_{k+1}\right|
$$

is called a gap. There are at most $n-2$ such gaps. In addition there are two special gaps, $\left[-A^{j-1}, A^{j-1}\right]$ where $A^{j} \leq\left|t_{2}\right| \leq A^{j+1}$, and $\left(-\infty,-A^{j_{*}+1}\right] \cup\left[A^{j_{*}+1}, \infty\right)$, where $j_{*}$ is the least integer such that $\left|t_{n}\right|<A^{j_{*}}$.

Two consecutive gaps are separated by a pair of "dyadic" intervals, symmetric with respect to the origin. In fact each of these "dyadic" intervals can contain at most $3 n$ intervals of the form $\left[A^{m}, A^{m+1}\right]$ 
or $\left[-A^{m+1},-A^{m}\right]$. The idea of the remainder of the proof is that such dyadic intervals are harmless since the contribution to $\int_{-\infty}^{\infty} f(x-$ $p(t)) d t / t$ arising from such an interval is clearly controlled by a constant times $M_{p} f(x)$, while on the gaps - where $p^{\prime}$ and $p$ (except at $0)$ have no zeros - one can try to change variables as in the proof of Theorem 2.1. However this is not entirely straightforward because of the nature of the cancellation in the problem.

We now indicate how to handle the contribution to $\int_{-\infty}^{\infty} f(x-$ $p(t)) d t / t$ arising from an (ordinary) gap; the minor changes of detail required for the special gaps are left to the reader. Suppose the gap is $\left[-A^{\ell},-A^{j}\right] \cup\left[A^{j}, A^{\ell}\right]$ with $\ell-j \geq 1$ and with $\left|t_{k}\right|<A^{j-1}<A^{\ell+1}<$ $\left|t_{k+1}\right|,(2 \leq k \leq n-1)$. (Note that there is a slight change of notation here.) Of course $A$ is chosen so that Lemma 2.5 is valid.

By part a) of Lemma 2.5,

$$
\begin{aligned}
\left|p\left(A^{\ell}\right)\right| & \geq\left(1-\frac{1}{A}\right)^{n-1} A^{\ell k} \prod_{m=k+1}^{n}\left|t_{m}\right| \\
& >\left(1+\frac{1}{A}\right)^{n-1} A^{j k} \prod_{m=k+1}^{n}\left|t_{m}\right| \\
& \geq\left|p\left(-A^{j}\right)\right|
\end{aligned}
$$

(if also $A>((A+1) /(A-1))^{n-1}$ ) and similarly $\left|p\left(-A^{\ell}\right)\right|>\left|p\left(A^{j}\right)\right|$. Thus the intervals $\left[\left|p\left(A^{j}\right)\right|,\left|p\left(A^{\ell}\right)\right|\right]$ and $\left[\left|p\left(-A^{j}\right)\right|,\left|p\left(-A^{\ell}\right)\right|\right]$ have a nonempty intersection $[a, b]$, say. Then, by Lemma 2.5.c), there is a unique $\alpha \in\left[A^{j}, A^{\ell}\right]$ such that $|p(\alpha)|=a$ and a unique $\beta>\alpha, \beta \in$ $\left[A^{j}, A^{\ell}\right]$ such that $|p(\beta)|=b$. Similarly there are unique $-\delta<-\gamma \in$ $\left[-A^{\ell},-A^{j}\right]$ such that $|p(-\gamma)|=a,|p(-\delta)|=b$. Observe that the set

$$
\left(\left[A^{j}, A^{\ell}\right] \backslash[\alpha, \beta]\right) \cup\left(\left[-A^{\ell},-A^{j}\right] \backslash[-\delta,-\gamma]\right)
$$

is the union of two intervals whose logarithmic measure is bounded above by an absolute constant. (This follows again by Lemma 2.5.a); we suggest the reader draw a picture.) Therefore the integral over this set is dominated by $M_{p} f(x)$.

We have thus reduced matters to estimating

$$
\int_{\alpha}^{\beta} f(x-p(t)) \frac{d t}{t}+\int_{-\delta}^{-\gamma} f(x-p(t)) \frac{d t}{t}
$$


We distinguish between two cases:

i) $p$ has the same sign on both intervals $[\alpha, \beta]$ and $[-\delta,-\gamma]$; say $p>0$,

ii) $p$ has opposite signs on the two intervals; say $p>0$ for $t>0$.

Case i). We observe that

$$
\int_{\alpha}^{\beta} \frac{p^{\prime}(t)}{p(t)} d t=-\int_{-\delta}^{-\gamma} \frac{p^{\prime}(t)}{p(t)} d t
$$

since $p(\alpha)=p(-\gamma)$ and $p(\beta)=p(-\delta)$. Thus it is enough to estimate two similar integrals separately, one of which is

$$
\int_{\alpha}^{\beta} f(x-p(t))\left(\frac{1}{t}-\frac{p^{\prime}(t)}{k p(t)}\right) d t
$$

where $\left|t_{k}\right|<A^{j-1}<A^{\ell+1}<\left|t_{k+1}\right|$.

Now, for $t \in[\alpha, \beta] \subseteq\left[A^{j}, A^{\ell}\right]$,

$$
\begin{aligned}
\left|\frac{1}{t}-\frac{p^{\prime}(t)}{k p(t)}\right| & =\left|\frac{1}{t}-\frac{1}{k} \sum_{m=1}^{n} \frac{1}{t-t_{m}}\right| \\
& \leq \frac{1}{k} \sum_{m=1}^{k}\left|\frac{1}{t}-\frac{1}{t-t_{m}}\right|+\frac{1}{k} \sum_{m=k+1}^{n} \frac{1}{\left|t-t_{m}\right|} \\
& =\frac{1}{k} \sum_{m=1}^{k} \frac{\left|t_{m}\right|}{|t|\left|t-t_{m}\right|}+\frac{1}{k} \sum_{m=k+1}^{n} \frac{1}{\left|t-t_{m}\right|} \\
& \leq \frac{c_{1} A^{j}}{t^{2}}+c_{2} A^{-\ell},
\end{aligned}
$$

where $c_{1}$ and $c_{2}$ depend upon $n$ and $A$.

Therefore

$$
\begin{aligned}
\left|\int_{\alpha}^{\beta} f(x-p(t))\left(\frac{1}{t}-\frac{p^{\prime}(t)}{k p(t)}\right) d t\right| \leq & c_{1} A^{j} \int_{A^{j}}^{\infty}|f(x-p(t))| \frac{d t}{t^{2}} \\
& +c_{2} A^{-\ell} \int_{0}^{A^{\ell}}|f(x-p(t))| d t \\
\leq & c_{3} M_{p} f(x) .
\end{aligned}
$$


Case ii). It is here that we finally use the cancellation in the operator. Indeed,

$$
\begin{aligned}
\int_{\alpha}^{\beta} & f(x-p(t)) \frac{d t}{t}+\int_{-\delta}^{-\gamma} f(x-p(t)) \frac{d t}{t} \\
= & \int_{\alpha}^{\beta} f(x-p(t))\left(\frac{1}{t}-\frac{p^{\prime}(t)}{k p(t)}\right) d t+\int_{-\delta}^{-\gamma} f(x-p(t))\left(\frac{1}{t}-\frac{p^{\prime}(t)}{k p(t)}\right) d t \\
& \quad+\frac{1}{k} \int f(x-p(t)) \frac{p^{\prime}(t)}{p(t)}\left(\chi_{[\alpha, \beta]}(t)+\chi_{[-\delta,-\gamma]}(t)\right) d t
\end{aligned}
$$

The first two integrals are treated exactly as in case i), while for the third we change variables separately on $[\alpha, \beta]$ and $[-\delta,-\gamma]$ to obtain

$$
\frac{1}{k} \int_{a \leq|u| \leq b} f(x-u) \frac{d u}{u},
$$

which is controlled by the maximal Hilbert transform as desired. This concludes the proof of Theorem 2.4.

\section{3. $\mathfrak{p}(x, t)$ as a polynomial in $t$.}

Let $\mathfrak{p}: \mathbb{R} \times \mathbb{R} \longrightarrow \mathbb{R}$ be a polynomial such that $\mathfrak{p}(x, 0)=x$. Let

$$
M_{\mathfrak{p}} f(x)=\sup _{h>0} \frac{1}{2 h} \int_{-h}^{h} f(\mathfrak{p}(x, t)) d t
$$

and

$$
H_{\mathfrak{p}} f(x)=\int_{-\infty}^{\infty} f(\mathfrak{p}(x, t)) \frac{d t}{t}
$$

be the maximal function and Hilbert transform respectively associated to $\mathfrak{p}$. We write

$$
\mathfrak{p}(x, t)=x+A_{1}(x) t+A_{2}(x) t^{2}+\cdots+A_{n}(x) t^{n},
$$

so that $\mathfrak{p}$ has degree at most $n$ as a polynomial in $t ; A_{1}, \ldots, A_{n}$ are for the moment arbitrary polynomial functions of $x$. 


\subsection{Results with no conditions on the coefficients.}

In view of the negative parts of Theorems 2.1 and 2.3, the only possible general positive result (with no conditions placed on the coefficients) is:

Theorem 3.1. For $\mathfrak{p}(x, t)$ an arbitrary polynomial of degree $n$ in $t$ such that $\mathfrak{p}(x, 0)=x$, the operators $M_{\mathfrak{p}}$ and $H_{\mathfrak{p}}$ are bounded on $L^{q}(\mathbb{R})$ for $q>n$ and are of restricted weak-type $n-n$.

This result is sharp in so far as for each $n$ there exists a $\mathfrak{p}$ of degree $n$ in $t$ as in the statement of the theorem with $M_{\mathfrak{p}}$ and $H_{\mathfrak{p}}$ unbounded on $L^{n}(\mathbb{R})$. Indeed, letting $\mathfrak{p}(x, t)=x(1-t)^{n}$, the proof of the sharpness of Theorems 2.1 and 2.3 applies here also. When $n=2$ we give below in Corollary 3.7 a complete analysis of the $L^{q}$ boundedness problem for each $\mathfrak{p}$.

\subsection{Many coefficients vanishing - the quadratic case.}

When all but one of the $A_{j}$ 's is identically zero and the remaining one is a completely arbitrary function of $x$, then $H_{\mathfrak{p}}$ and $M_{\mathfrak{p}}$ are dominated by the standard Hilbert transform and maximal function respectively and so are of weak-type 1-1 and are $L^{q}$ bounded, $1<q<\infty$. (If $j$ is even and $A_{j}(x)$ is the nonzero coefficient, then $H_{\mathfrak{p}} \equiv 0$.)

The situation when all but two of the $A_{j}$ 's are identically zero is already considerably more complicated; the first special case of this is

$$
\mathfrak{p}(x, t)=x+A_{1}(x) t+A_{2}(x) t^{2}
$$

corresponding to polynomials of degree 2 in $t$.

In Theorem 3.2 we give an analysis of this quadratic case. We have carried out a similar but much lengthier analysis of the cubic case which we do not propose to present here; the interested reader is invited to contact one of the authors for details. (We estimate that merely a statement of the result would fill several printed pages and so we have chosen not to unecessarily burden the reader at this moment.)

We set up some notation. Let $p$ and $q$ be arbitrary $C^{1}$ functions of $x$. We write $A_{1}=p$ and $A_{2}=q$ so that

$$
\mathfrak{p}(x, t)=x+t p(x)+t^{2} q(x) .
$$


We let $\Delta(x)=p^{2}(x)-4 x q(x)$ be the discriminant of $\mathfrak{p}(x, \cdot)$ as a quadratic in $t$, and when $q(x) \neq 0$ we let $\psi(x)=\Delta(x) / 4 q(x)$. We shall require $\psi$ to have some smoothness. It turns out that the critical points of $\psi$ play a decisive role. We say that $\psi$ has a monotonic critical point at $\pm \infty$ if $\lim _{x \rightarrow \pm \infty} \psi^{\prime}(x)=0$ and $\psi^{\prime}$ is single signed as $x \longrightarrow \pm \infty$. We say that $\psi$ has a critical point of finite order $k \geq 2$ at $x_{0} \in \mathbb{R}$ if

$$
\psi(x)=\psi\left(x_{0}\right)+\delta\left(x-x_{0}\right)^{k}+O\left(\left|x-x_{0}\right|^{k+1}\right)
$$

with $\delta \neq 0$.

Theorem 3.2. With the notation as above, let $\mathfrak{p}(x, t)=x+t p(x)+$ $t^{2} q(x)$ with $p, q \in C^{1}$ such that $Z_{q}=\{q(x)=0\}$ is finite.

i) If $\left|\psi^{\prime}\right|$ is bounded below on $\mathbb{R} \backslash Z_{q}$ then $M_{\mathfrak{p}}$ and $H_{\mathfrak{p}}$ are of weaktype 1-1 and are bounded on $L^{r}, 1<r<\infty$.

ii) If $\left|\psi^{\prime}\right|$ is bounded below at $\pm \infty$ and near $Z_{q}$, if $\psi$ has finitely many critical points of finite order at each of which $\psi(x)+x$ is nonzero, then $M_{\mathfrak{p}}$ and $H_{\mathfrak{p}}$ are bounded on $L^{r}$ if and only if $r \geq 2(k-1) / k$, where $k$ is the maximum of the orders of the critical points. When $k=2$ this must be modified to read as $M_{\mathfrak{p}}$ and $H_{\mathfrak{p}}$ are of weak-type 1-1 and bounded on $L^{r}, 1<r<\infty$.

iii) If either

a) $\psi$ has a monotonic critical point at $\pm \infty$, or

b) $\psi$ has a critical point of finite order at $x_{0}$ such that

$$
\psi\left(x_{0}\right)+x_{0}=0
$$

then $M_{\mathfrak{p}}$ and $H_{\mathfrak{p}}$ are unbounded on $L^{2}$, and bounded on $L^{r}$ for $r>2$.

Before proving this theorem we first give some lemmas.

\section{Lemma 3.3.}

$\sup _{\substack{p, q \in \mathbb{R} \backslash\{0\} \\ h>0}} \frac{1}{h} \int_{[h, 2 h] \cap\{|t+p /(2 q)| \geq|p| /(4|q|)\}}\left|f\left(x+p t+q t^{2}\right)\right| d t \leq C M f(x)$,

where $M f$ is the ordinary Hardy-Littlewood maximal function of $f$. 
Proof. By scaling it is enough to take $h=1$. Assume without loss of generality that $q>0$. We split the integral into two pieces, the first over $|p| /(4 q) \leq|t+p /(2 q)| \leq 10|p| / q$, and the second over $|t+p /(2 q)| \geq$ $10|p| / q$. Let $u=u(t)=p t+q t^{2}$; then

$$
\left|u^{\prime}(t)\right|=|p+2 q t|=2 q\left|t+\frac{p}{2 q}\right| \approx|p|
$$

in the first case and

$$
\left|u^{\prime}(t)\right|=|p+2 q t|=2 q\left|t+\frac{p}{2 q}\right| \approx q|t| \approx q^{1 / 2} u^{1 / 2}
$$

in the second case. Thus,

$$
\begin{aligned}
\int_{[1,2] \cap\{|p| /(4 q) \leq|t+p /(2 q)| \leq 10|p| / q\}}\left|f\left(x+p t+q t^{2}\right)\right| d t \\
\quad \leq \int_{\left\{|u| \leq C\left|p^{2}\right| / q\right\} \cap u[1,2]}|f(x+u)| \frac{d u}{|p|} \\
\quad=\left|\frac{p}{q}\right|\left|\frac{q}{p^{2}}\right| \int_{\left\{|u| \leq C\left|p^{2}\right| / q\right\} \cap u[1,2]}|f(x+u)| d u \\
\leq \operatorname{CMf}(x),
\end{aligned}
$$

since if $1 \leq t \leq 2$, we get a nonzero contribution only when $|p| /|q| \approx C$. For the second piece

$$
\begin{aligned}
\int_{[1,2] \cap\{|t+p /(2 q)| \geq 10|p| / q\}} \mid & f\left(x+p t+q t^{2}\right) \mid d t \\
& \leq C \int_{u \sim q}|f(x+u)| \frac{d u}{q^{1 / 2} u^{1 / 2}} \\
& \leq C M f(x)
\end{aligned}
$$

since if $1 \leq t \leq 2$ and $|t+p /(2 q)| \geq 10|p| / q$ then $|u(t)| \approx q t^{2} \approx q$.

\section{Corollary 3.4.}

$$
\sup _{\substack{p, q \in \mathbb{R} \backslash\{0\} \\ h>0}} \frac{1}{h} \int_{[-h, h] \cap\{|t+p /(2 q)| \geq|p| / 4|q|\}}\left|f\left(x+p t+q t^{2}\right)\right| d t \leq C M f(x) .
$$


Proof. Break up $[-h, h]$ into dyadic intervals $\pm\left[2^{-k} h, 2^{-k+1} h\right]$ and use Lemma 3.3 on each to obtain a convergent geometric series.

Thus for the maximal function problem, all we need consider is

$$
\tilde{M}_{\mathfrak{p}} f(x)=\sup _{h>0} \frac{1}{h} \int_{\mathcal{H}}\left|f\left(x+p(x) t+q(x) t^{2}\right)\right| d t,
$$

where

$$
\mathcal{H}=[-h, h] \cap\left\{\left|t+\frac{p(x)}{2 q(x)}\right| \leq \frac{|p(x)|}{4|q(x)|}\right\} .
$$

Now when $p(x)$ or $q(x)$ (or both) are zero, $M_{\mathfrak{p}} f(x) \leq C M f(x)$ and $\left|H_{\mathfrak{p}} f(x)\right| \leq C|H f(x)|$, so that we may assume here and in what follows that we need consider only $x$ with $p(x), q(x) \neq 0$. By virtue of Theorem 2.4, we have

$$
\left|H_{\mathfrak{p}} f(x)\right| \leq A_{2} M_{\mathfrak{p}} f(x)+B_{2} H^{*} f(x) \leq C\left(\tilde{M}_{\mathfrak{p}} f(x)+M f(x)+H^{*} f(x)\right)
$$

and so to control the Hilbert transform we again only need consider $\tilde{M}_{\mathfrak{p}} f(x)$. Furthermore it is easily seen (using arguments from Lemma 3.3 and Theorem 2.4) that

$$
\left|H_{\mathfrak{p}} f(x)-\tilde{H}_{\mathfrak{p}} f(x)\right| \leq C\left(M f(x)+H^{*} f(x)\right),
$$

where

$$
\text { (4) } \quad \tilde{H}_{\mathfrak{p}} f(x)=\int_{|t+p(x) /(2 q(x))| \leq|p(x)| /(4|q(x)|)} f\left(x+p(x) t+q(x) t^{2}\right) \frac{d t}{t} \text {. }
$$

Since for each fixed $x$, the integral in (4) is over a dyadic interval, there is no further cancellation in the operator $\tilde{H}_{\mathfrak{p}}$ and indeed $\tilde{H}_{\mathfrak{p}}$ is essentially a contribution to $\tilde{M}_{\mathfrak{p}}$ where $h$ takes the value $2|p(x)| /|q(x)|$. On the other hand this value of $h$ is the only interesting one contributing to $\tilde{M}_{p}$, and so the operators $\tilde{M}_{\mathfrak{p}}$ and $\tilde{H}_{\mathfrak{p}}$ are both essentially equivalent to

$$
\begin{aligned}
& R_{\mathfrak{p}} f(x) \\
& \quad=\left|\frac{q(x)}{p(x)}\right| \\
& \quad \int_{|t+p(x) /(2 q(x))| \leq|p(x)| / 4|q(x)|} f\left(x+p(x) t+q(x) t^{2}\right) d t,
\end{aligned}
$$


which therefore governs the behaviour of both $M_{\mathfrak{p}}$ and $H_{\mathfrak{p}}$.

At this point it is appropriate to comment upon the simple averaging operator

$$
S_{\mathfrak{p}} f(x)=\int_{1}^{2} f\left(x+p(x) t+q(x) t^{2}\right) d t .
$$

Clearly $S_{\mathfrak{p}}$ is dominated by $M_{\mathfrak{p}}$, and if $H_{\mathfrak{p}}$ has certain boundedness property, so does $S_{\mathfrak{p}}$ (see for example [CG].) On the other hand, making the change of variables $t=u p(x) / q(x)$ in (5) gives

$$
R_{\mathfrak{p}} f(x)=\int_{|u+1 / 2| \leq 1 / 4} f\left(x+\tilde{p}(x) u+\tilde{q}(x) u^{2}\right) d u,
$$

where $\tilde{p}(x)=p^{2}(x) / q(x)$ and $\tilde{q}(x)=p^{2}(x) / q(x)$ also. Thus $R_{\mathfrak{q}}$ arises essentially as $S_{\widetilde{\mathfrak{p}}}$ where

$$
\widetilde{\mathfrak{p}}(x, t)=x+\frac{p^{2}(x)}{q(x)} t+\frac{p^{2}(x)}{q(x)} t^{2} .
$$

Thus positive results for $S_{\widetilde{\mathfrak{p}}}$ imply corresponding ones for $S_{\mathfrak{p}}$ although there is no formal invariance property from which this follows. Notice that if we define $\tilde{\Delta}=\tilde{p}^{2}-4 x \tilde{q}(x)$ and $\tilde{\psi}=\tilde{\Delta} / 4 \tilde{q}$, then $\tilde{\psi}=\psi$ and $\tilde{p}^{2} / 4 \tilde{q}=p^{2} / 4 q$; that is, the quantities arising in the statement of Theorem 3.2 remain invariant, which is natural since the basic problems for $M_{\mathfrak{p}}$ and $H_{\mathfrak{p}}$ are invariant under

$$
(p, q) \longmapsto\left(p(x) h(x), q(x) h(x)^{2}\right)=(\widetilde{\widetilde{p}}, \widetilde{\widetilde{q}})
$$

for any $h(x) \neq 0$. Indeed, the basic problem for $M_{\mathfrak{p}}$ is equivalent to that for $S_{\widetilde{\mathfrak{p}}}$ with arbitrary $h(x)$, as can be seen by linearising $M_{\mathfrak{p}}$ with $h(x)$.

Performing the further changes of variables $u=v-1 / 2$ and then $v=\{s / \tilde{p}(x)\}^{1 / 2}$ (assuming that $\tilde{p}(x)>0$ without loss of generality) yields in $(7)$

$$
T_{\tilde{\mathfrak{p}}} f(x)=\frac{1}{\tilde{p}(x)^{1 / 2}} \int_{0 \leq s \leq \tilde{p}(x)} f(s-\tilde{\psi}(x)) \frac{d s}{s^{1 / 2}}
$$

as the operator determining the behaviour of $M_{\mathfrak{p}}$ and $H_{\mathfrak{p}}$. 
Lemma 3.5. For $0 \leq x \leq 1, \alpha \geq 2$ and $\beta \geq 0$ define

$$
T_{\alpha, \beta} f(x)=\frac{1}{x^{\beta / 2}} \int_{0}^{x^{\beta}} f\left(s-x^{\alpha}\right) \frac{d s}{s^{1 / 2}} .
$$

Then for $p<\infty, T_{\alpha, \beta}$ is bounded from $L^{p}(\mathbb{R})$ to $L^{p}(0,1)$.

i) If $\beta=0$ for $p \geq 2(\alpha-1) / \alpha$, except when $\alpha=2$, in which case for $p>1$; moreover $T_{2,0}$ is of weak-type $1-1$,

ii) if $0<\beta<1$ for $p \geq 2(\alpha-1) /(\alpha-\beta)$,

iii) if $\beta=1$ for $p>2$.

In all other cases, or if $\beta>1, T_{\alpha, \beta}$ is unbounded.

Proof. Let $\psi(x)=x^{\alpha}$. Then

$$
\begin{aligned}
\int_{0}^{1}\left|T_{\alpha, \beta} f(x)\right|^{p} d x & \leq \int_{0}^{1} \frac{1}{x^{p \beta / 2}}\left|I_{1 / 2} f(\psi(x))\right|^{p} d x \\
& =\int_{0}^{1} \frac{1}{\psi^{-1}(u)^{p \beta / 2}}\left|I_{1 / 2} f(u)\right|^{p} \frac{d u}{\psi^{\prime}\left(\psi^{-1}(u)\right)}
\end{aligned}
$$

where $I_{1 / 2}$ is the standard fractional integral of order $1 / 2$. Now $\psi^{\prime}(x)=$ $\alpha x^{\alpha-1}$ and $\psi^{-1}(u)=u^{1 / \alpha}$. So

$$
\psi^{-1}(u)^{-p \beta / 2} \psi^{\prime}\left(\psi^{-1}(u)\right)^{-1}=\alpha^{-1} u^{-p \beta /(2 \alpha)} u^{-1+1 / \alpha}
$$

which belongs to the space $L^{r, \infty}(0,1), 1 \leq r \leq \infty$, precisely when $1 \leq r \leq 2 \alpha /(2 \alpha+p \beta-2)$. Thus

$$
\int_{0}^{1}\left|T_{\alpha, \beta} f(x)\right|^{p} d x \leq C\left\|\left|I_{1 / 2} f\right|^{p}\right\|_{L^{r^{\prime}, 1}}=C\left\|I_{1 / 2} f\right\|_{L^{r^{\prime} p, p}}^{p},
$$

provided $1<r=2 \alpha /(2 \alpha+p \beta-2)$. Now, by the Marcinkiewicz interpolation theorem (see [StW]), $I_{1 / 2}: L^{p, p} \longrightarrow L^{q, p}$ for $1 / q=1 / p-$ $1 / 2,1 / 2<1 / p<1$, and so $T_{\alpha, \beta}$ is bounded on $L^{p}$ if $1 /\left(r^{\prime} p\right)=1 / p-1 / 2$, i.e. $1 /(p r)=1 / 2$, i.e. $p=2(\alpha-1) /(\alpha-\beta)$ if this number lies in $(1,2)$, which when $\beta=0$ is when $\alpha>2$, when $\beta \in(0,1)$ is for all $\alpha \geq 2$ and for $\beta=1$ does not occur. We have thus proved the positive assertions of the lemma with the exception of the case $\alpha=2, \beta=0$ and $\alpha$ arbitrary, $\beta=1$. The results for $p>1$ and $p>2$ respectively follow from 
(nonsharp) $L^{p} \longrightarrow L^{q}$ mapping properties of $I_{1 / 2}$, while the weak-type 1-1 result for $T_{2,0}$ follows since

$$
\begin{aligned}
\left|\left\{x:\left|T_{2,0} f(x)\right|>\lambda\right\}\right| & =\int \chi_{\left\{x:\left|T_{2,0} f(x)\right|>\lambda\right\}} d x \\
& =\int \chi_{\left\{u:\left|I_{1 / 2} f(u)\right|>\lambda\right\}} \frac{d u}{\psi^{\prime}\left(\psi^{-1}(u)\right)} \\
& \leq\left\|\frac{1}{u^{1 / 2}}\right\|_{L^{2, \infty}}\left\|\chi_{\left\{u:\left|I_{1 / 2} f(u)\right|>\lambda\right\}}\right\|_{L^{2,1}} \\
& =C\left|\left\{u:\left|I_{1 / 2} f(u)\right|>\lambda\right\}\right|^{1 / 2} \\
& \leq \frac{C\|f\|_{1}}{\lambda},
\end{aligned}
$$

as $I_{1 / 2}: L^{1} \longrightarrow L^{2, \infty}$.

$T_{2,0}$ is clearly not bounded on $L^{1}$ (test on $f=\delta_{0}$ ). For the other necessary conditions, first let $f=\chi_{(-\delta, 0)}$. Then, for $x^{\alpha}<\delta$,

$$
T_{\alpha, \beta} f(x)=\frac{1}{x^{\beta / 2}} \int_{0}^{x^{\alpha \vee \beta}} \frac{d s}{s^{1 / 2}}= \begin{cases}C, & \beta \geq \alpha, \\ C x^{(\alpha-\beta) / 2}, & \beta \leq \alpha,\end{cases}
$$

and so $T_{\alpha, \beta} f$ has $L^{p}$ norm bounded below by

$$
\begin{cases}\delta^{1 /(\alpha p)}, & \beta \geq \alpha \\ \delta^{(\alpha-\beta) /(2 \alpha)+1 /(\alpha p)}, & \beta \leq \alpha .\end{cases}
$$

Hence, when $\beta \geq \alpha, \alpha$ is forced to be at most 1, (violating our assumption $\alpha \geq 2$ ) and when $\beta \leq \alpha$, we must have

$$
\frac{\alpha-\beta}{2 \alpha}+\frac{1}{\alpha p} \geq \frac{1}{p}
$$

i.e. $p \geq 2(\alpha-1) /(\alpha-\beta)$. Secondly, to see $\beta \leq 1$ is necessary, assume $\beta<\alpha$ (for when $\beta \geq \alpha$ we have already seen there are no $p$ for which $T_{\alpha, \beta}$ is bounded on $L^{p}$ ), and observe that, for $f \geq 0$,

$T_{\alpha, \beta} f(x) \geq \frac{1}{x^{\beta / 2}} \int_{x^{\alpha}}^{x^{\beta}} f\left(s-x^{\alpha}\right) \frac{d s}{s^{1 / 2}}=\frac{1}{x^{\beta / 2}} \int_{0}^{x^{\beta}-x^{\alpha}} f(s) \frac{d s}{\left(s+x^{\alpha}\right)^{1 / 2}}$.

Now set $f=\chi_{(0, \delta)}$ and observe that for $x^{\beta}<\delta$,

$$
T_{\alpha, \beta} f(x)=\frac{1}{x^{\beta / 2}} \int_{0}^{x^{\beta}-x^{\alpha}} \frac{d s}{\left(s+x^{\alpha}\right)^{1 / 2}}=\frac{1}{x^{\beta / 2}} \int_{x^{\alpha}}^{x^{\beta}} \frac{d s}{s^{1 / 2}} \approx C .
$$


Thus $\left\|T_{\alpha, \beta} f\right\|_{p} \geq c \delta^{1 / \beta p}$, while $\|f\|_{p} \sim \delta^{1 / p}$. Hence indeed $\beta \leq 1$. Finally, to see that $T_{\alpha, 1}$ is not bounded on $L^{2}$, (nor indeed of weaktype 2-2), let

$$
f(s)=\frac{\chi_{(0,1 / 2)}(s)}{s^{1 / 2} \log \left(\frac{1}{s}\right)} \in L^{2} .
$$

Then

$$
\begin{aligned}
T_{\alpha, 1} f(x) & \geq \frac{1}{x^{1 / 2}} \int_{0}^{x-x^{\alpha}} \frac{1}{s^{1 / 2} \log \left(\frac{1}{s}\right)} \frac{d s}{\left(s+x^{\alpha}\right)^{1 / 2}} \\
& =\frac{1}{x^{1 / 2}} \int_{x^{\alpha}}^{x} \frac{1}{\left(s-x^{\alpha}\right)^{1 / 2} \log \left(\frac{1}{s-x^{\alpha}}\right)} \frac{d s}{s^{1 / 2}} \\
& \geq \frac{1}{x^{1 / 2}} \int_{x^{\alpha}}^{x} \frac{d s}{s \log \left(\frac{1}{s}\right)} \\
& \geq \frac{c}{x^{1 / 2}}
\end{aligned}
$$

which is not in $L^{2}$.

$T_{\alpha, 1}$ is also of restricted weak-type $2-2$. This follows from the proof of Theorem 3.2.

Lemma 3.6. Suppose $\psi^{\prime}(x) \longrightarrow 0$ as $x \longrightarrow \infty$, and that $\psi^{\prime}(x) \geq 0$ for sufficiently large $x$. Let

$$
T_{\psi} f(x)=\frac{1}{x^{1 / 2}} \int_{0}^{x} f(t-\psi(x)) \frac{d t}{t^{1 / 2}} .
$$

Then $T_{\psi}$ is unbounded from $L^{2}(\mathbb{R})$ to $L^{2}((0,1))$.

Proof. We may assume that $\psi(x)>0$ for sufficiently large $x$. Then

$$
\begin{aligned}
\frac{1}{x^{1 / 2}} \int_{0}^{x} f(t-\psi(x)) \frac{d t}{t^{1 / 2}} & =\frac{1}{x^{1 / 2}} \int_{-\psi(x)}^{x-\psi(x)} f(s) \frac{d s}{(s+\psi(x))^{1 / 2}} \\
& \geq \frac{1}{x^{1 / 2}} \int_{0}^{x-\psi(x)} f(s) \frac{d s}{(s+\psi(x))^{1 / 2}},
\end{aligned}
$$


for $f \geq 0$. Let $f=\chi_{(0, A)}$ with $A$ large. Then

$$
T_{\psi} f(x) \geq \frac{1}{x^{1 / 2}} \int_{0}^{A} \frac{d s}{(s+\psi(x))^{1 / 2}}, \quad \text { for } x \gg A
$$

since $\psi^{\prime} \longrightarrow 0$ implies

$$
\frac{\psi(x)}{x}=\frac{\psi\left(x_{0}\right)}{x}+\frac{x-x_{0}}{x} \frac{1}{x-x_{0}} \int_{x_{0}}^{x} \psi^{\prime}(u) d u
$$

goes to zero as $x \longrightarrow \infty$. Hence, for such $x$,

$$
T_{\psi} f(x) \geq \frac{1}{x^{1 / 2}} \int_{\psi(x)}^{A+\psi(x)} \frac{d s}{s^{1 / 2}} \sim \frac{A}{x^{1 / 2}(A+\psi(x))^{1 / 2}}
$$

Therefore, for appropriate constants $C_{1}$ and $C_{2}$,

$$
\left(\int\left|T_{\psi} f(x)\right|^{2} d x\right)^{1 / 2} \geq C A^{1 / 2}\left(\int_{\left\{x: x \geq C_{1} A, \psi(x) \leq C_{2} A\right\}} \frac{d x}{x}\right)^{1 / 2} \gg A^{1 / 2}
$$

while $\|f\|_{2} \sim A^{1 / 2}$.

Proof of Theorem 3.2. By the discussion between Corollary 3.4 and Lemma 3.5, it is sufficient to study the operators given by (8), that is

$$
T_{\widetilde{\mathfrak{p}}} f(x)=\frac{1}{\tilde{p}(x)^{1 / 2}} \int_{0 \leq s \leq \tilde{p}(x)} f(s-\tilde{\psi}(x)) \frac{d s}{s^{1 / 2}}
$$

where $\tilde{p}=\tilde{q}=p^{2} / q, \tilde{\psi}=\tilde{\Delta} / 4 \tilde{q}, \tilde{\Delta}=\tilde{p}^{2}(x)-4 x \tilde{q}(x)$, so that $\psi(x)=$ $\tilde{\psi}(x)=\tilde{p}(x) / 4-x$. Thus $\psi(x)+x$ vanishes if and only if $\tilde{p}(x)$ vanishes. (Of course it is neighbourhoods of such points rather than the points themselves which concern us in obtaining $L^{r}$ estimates.) We change notation; we replace $\tilde{\psi}$ by $\psi, \tilde{p}$ by $p$ and $\widetilde{\mathfrak{p}}$ by $\mathfrak{p}$.

i) Let us first assume $\psi^{\prime} \geq C>0$ on $\mathbb{R}$. Then, since we always have $\left|T_{\mathfrak{p}} f(x)\right| \leq C M f(\psi(x))$ where $M$ is the ordinary Hardy-Littlewood 
maximal function, we can write

$$
\begin{aligned}
\left|\left\{x:\left|T_{\mathfrak{p}} f(x)\right|>\lambda\right\}\right| & \leq\left|\left\{x: M f(\psi(x))>\frac{\lambda}{C}\right\}\right| \\
& =\int \chi_{\{x: M f(\psi(x))>\lambda / C\}} d x \\
& =\int \chi_{\{u: M f(u)>\lambda / C\}} \frac{d u}{\psi^{\prime}\left(\psi^{-1}(u)\right)} \\
& \leq c^{\prime}\left|\left\{u: M f(u)>\frac{\lambda}{C}\right\}\right| \\
& \leq c^{\prime \prime} \frac{\|f\|_{1}}{\lambda} .
\end{aligned}
$$

Notice that the same argument controls the behaviour of $T_{\mathfrak{p}} f(x)$ on any interval of $x$ upon which $\left|\psi^{\prime}\right|$ is bounded below.

ii) By the proof of i) it is enough to consider the behaviour of $T_{\mathfrak{p}}$ near a critical point, say 0 , of maximal order $k$. Now $p(0) \neq 0$ implies that by taking a small enough neighbourhood of zero, we can assume $p(x) \sim \varepsilon>0$. After a translation of $f$ we can assume, then, that

$$
T_{\mathfrak{p}} f(x) \approx \int_{0}^{\varepsilon} f\left(s-\delta x^{k}+O\left(x^{k+1}\right)\right) \frac{d s}{s^{1 / 2}},
$$

which is essentially the situation of Lemma 3.5 , case $\beta=0, \alpha=k$. (The proof of Lemma 3.5 can be easily modified to give the variant required here.)

iii) Suppose first that $\psi$ has a monotonic critical point at $\infty$. Then $\lim _{x \rightarrow \infty} p^{\prime}(x)=4$ and thus $p(x) \sim x$ for large $x$. So in this case,

$$
T_{\mathfrak{p}} f(x) \sim \frac{1}{x^{1 / 2}} \int_{0}^{x} f(s-\psi(x)) \frac{d s}{s^{1 / 2}},
$$

which is unbounded on $L^{2}$ by Lemma 3.6.

If $\psi$ has a critical point of finite order at $x_{0}$, then $0=\psi^{\prime}\left(x_{0}\right)=$ $p^{\prime}\left(x_{0}\right) / 4-1$ which implies that

$$
p(x)=p\left(x_{0}\right)+4\left(x-x_{0}\right)+O\left(x-x_{0}\right)^{2}
$$

near $x_{0}$. Assuming that $x_{0}=0$, and making a translation of $f$, we have

$$
T_{\mathfrak{p}} f(x) \sim \frac{1}{x^{1 / 2}} \int_{0}^{x} f(s-\psi(x)) \frac{d s}{s^{1 / 2}},
$$


which is the case $\beta=1$ of Lemma 3.5. Thus $T_{\mathfrak{p}}$ is unbounded on $L^{2}$ in this case too.

Corollary 3.7. Let $p(x)$ and $q(x)$ be polynomials in $x, \mathfrak{p}(x, t)=x+$ t $p(x)+t^{2} q(x), \Delta(x)=p^{2}(x)-4 x q(x)$ and $\psi=\Delta /(4 q)$.

i) If $\operatorname{deg} \Delta>\operatorname{deg} q$ and $\psi$ has no critical points then $M_{p}$ and $H_{p}$ are of weak-type 1-1 and bounded on $L^{r}, 1<r<\infty$.

ii) If $\operatorname{deg} \Delta>\operatorname{deg} q, p^{2} /(4 q)$ does not vanish at any of the critical points of $\psi$, the largest of the orders of which is $k$, then $M_{\mathfrak{p}}$ and $H_{\mathfrak{p}}$ are bounded on $L^{r}$ if and only if $r \geq 2(k-1) / k$, except when $k=2$, in which case they are of weak-type 1-1 and bounded on $L^{r}, 1<r<\infty$.

iii) If $\operatorname{deg} \Delta>\operatorname{deg} q$ and $p^{2} /(4 q)$ vanishes at some critical point of $\psi$, if $\operatorname{deg} \Delta \leq \operatorname{deg} q$, or if $\Delta \equiv 0$, then $M_{\mathfrak{p}}$ and $H_{\mathfrak{p}}$ are unbounded on $L^{2}$.

Proof. When $\psi \neq 0, \psi^{\prime}$ vanishes at infinity if and only if $\operatorname{deg} \Delta \leq$ $\operatorname{deg} q$; when $\operatorname{deg} \Delta>\operatorname{deg} q, \psi^{\prime}$ is bounded below at infinity. Moreover $\psi^{\prime}$ is bounded below near $Z_{q}$ anyway. The result now follows from Theorem 3.2.

\subsection{Constant coefficients.}

When each of the $A$ 's is constant, then $H_{\mathfrak{p}}$ and $M_{\mathfrak{p}}$ are bounded on $L^{q}(\mathbb{R}), 1<q<\infty$, and are of weak-type 1-1. Moreover when $q>1$ the bounds may be taken to be independent of the $A$ 's. This latter statement for $H_{\mathfrak{p}}$ follows trivially from Theorem 2.3; for both $H_{\mathfrak{p}}$ and $M_{\mathfrak{p}}$ it is also a special case of [S2, Chapter XI, Section 2, Propositions 1 and 2]. However since the method of [S2] involves lifting to a higher dimensional setting $\mathbb{R}^{k}, k \geq 2$, where the lifted operators are now associated to curves in $\mathbb{R}^{k}$, the weak-type 1-1 estimate does not follow. We now present in Theorem 3.9 the result that the weaktype 1-1 bounds of $H_{\mathfrak{p}}$ and $M_{\mathfrak{p}}$ may be taken to be independent of the coefficients, and depend only on the degree. The following lemma is closely related to Lemma 3.3. It is also useful in examining higher degree analogues of Theorem 3.2.

Lemma 3.8. Let $p$ be a real polynomial of degree at most $n$, with $p(0)=0$ and leading coefficient 1 . Let $G$ be the union of the gaps of $p$ 
as in Section 2 above. Then

$$
\sup _{h>0}\left|\frac{1}{h} \int_{[h, 2 h] \cap G} f(x-p(t)) d t\right| \leq C_{n} M f(x),
$$

where $C_{n}$ depends only upon $n$ and $M f$ is the ordinary Hardy-Littlewood maximal function of $f$.

Proof. By scaling we may assume $h=1$. (Note that $J$ is a gap for $p$ if and only if $J / h$ is a gap for $\left.h^{-n} p(h \cdot)\right)$. By Lemma 2.5 we may change variables to obtain

$$
\begin{aligned}
\left|\int_{[1,2] \cap G} f(x-p(t)) d t\right| & =\left|\int_{p([1,2] \cap G)} f(x-u) \frac{d u}{\left|p^{\prime}\left(p^{-1}(u)\right)\right|}\right| \\
& \leq \frac{C}{\left|p^{\prime}\left(t_{0}\right)\right|} \int_{-\left|p\left(t_{1}\right)\right|}^{\left|p\left(t_{1}\right)\right|}|f(x-u)| d u,
\end{aligned}
$$

where $|p|$ attains its maximum on $[1,2] \cap G$ at $t_{1}$ and $\left|p^{\prime}\right|$ attains its minimum on $[1,2] \cap G$ at $t_{0}$. Now $\left|p\left(t_{1}\right)\right| \leq C\left|p\left(t_{0}\right)\right|$ by Lemma 2.5.a), and by Lemma 2.5.b), $\left|p\left(t_{0}\right)\right| \leq 2 \varepsilon_{0}(n)^{-1}\left|p^{\prime}\left(t_{0}\right)\right|$; so $\left|p\left(t_{1}\right)\right| \leq C\left|p^{\prime}\left(t_{0}\right)\right|$. Thus the integral above is dominated, independently of the coefficients of $p$, by the Hardy-Littlewood maximal function of $f$.

Theorem 3.9. Let $\mathfrak{p}(x, t)=x+\sum_{j=1}^{n} A_{j} t^{j}$ with $A_{j}$ constants. Then there exists $C(n)$ depending only upon $n$ and not on $\left\{A_{j}\right\}$ such that

$$
\left|\left\{x: M_{\mathfrak{p}} f(x)>\alpha\right\}\right| \leq C(n) \frac{\|f\|_{1}}{\alpha}
$$

and

$$
\left|\left\{x:\left|H_{\mathfrak{p}} f(x)\right|>\alpha\right\}\right| \leq C(n) \frac{\|f\|_{1}}{\alpha} .
$$

Proof. By Theorem 2.4 it is enough to prove the estimate for $M_{\mathfrak{p}}$. Let $p(t)=\sum_{j=1}^{n} A_{j} t^{j}$. Without loss of generality, assume $A_{n}=1$. It is enough to obtain the weak-type estimate for

$$
\sup _{k \in \mathbb{Z}}\left|\frac{1}{2^{k}} \int_{\left[2^{k}, 2^{k+1}\right]} f(x-p(t)) d t\right| .
$$

For all except boundedly may $k$ (with the bound depending only upon $n$ ) we can use Lemma 3.8 to dominate the integrals by $M f(x)$. The 
remaining $k$ 's correspond to a bounded number of finite measures of mass 1 and hence play no role.

It is interesting to note that one may also prove the quadratic case of Theorem 3.9 by dominating $M_{\mathfrak{p}} f(x)$ pointwise by $M f(x)+M f(x \pm$ $\left.p\left(t_{x}\right)\right)$ where $t_{x}$ is the critical point of $p$. The proof proceeds along the lines of that of Theorem 3.2, uses Lemma 3.3 and dominates $T_{\tilde{\mathfrak{p}}} f(x)$ by $M f\left(x \pm p\left(t_{x}\right)\right)$. It also suggests that it is really the gaps of $p$ which are also gaps of $p^{\prime}$ which are crucial in Lemma 3.8.

\section{References.}

[CCW] Carbery, A., Christ, M., Wright, J., Multidimensional van der Corput and sublevel set estimates. In preparation.

[CG] Carbery, A., Gillespie, T. A., In preparation.

[CWW1] Carbery, A., Wainger, S., Wright, J., The Hilbert transform and maximal function along flat curves in the Heisenberg group. J. Amer. Math. Soc. 8 (1995), 141-179.

[CWW2] Carbery, A., Wainger, S., Wright, J., Hilbert transforms and maximal functions along variable flat plane curves. J. Fourier Anal. Applications. Kahane Special Issue (1995), 119-139.

[Ch] Christ, M., Hilbert transforms along curves: I, Nilpotent Groups. Ann. of Math. 122 (1985), 575-596.

[deG] Guzmán, M. de, Real Variable Methods in Fourier Analysis. North Holland Mathematics Studies, 1981.

[HP] Hu, Y., Pan, Y., Boundedness of oscillatory singular integrals on Hardy spaces. Arkiv. Math. 30 (1992), 311-320.

[MR] Marletta, G., Ricci, F., Two-parameter maximal functions associated to homogeneous surfaces in $\mathbb{R}^{n}$. Preprint.

[PS1] Phong, D. H., Stein, E. M., Hilbert integrals, singular integrals and Radon transforms I. Acta Math. 157 (1986), 99-157.

[PS2] Phong, D. H., Stein, E. M., Oscillatory integrals with polynomial phases. Invent. Math. 110 (1992), 39-62.

[RS1] Ricci, F., Stein, E. M., Harmonic analysis on nilpotent groups and singular integrals I: Oscillatory integrals. J. Funct. Anal. 73 (1987), 179-194.

[RS2] Ricci, F., Stein, E. M., Harmonic analysis on nilpotent groups and singular integrals II: Singular kernels supported on submanifolds. J. Funct. Anal. 78 (1988), 56-84. 
[S1] Stein, E. M., Singular Integrals and Differentiability Properties of Functions. Princeton University Press, 1970.

[S2] Stein, E. M., Harmonic Analysis: Real Variable Methods, Orthogonality and Oscillatory Integrals. Princeton University Press, 1993.

[SW] Stein, E. M., Wainger, S., Problems in harmonic analysis related to curvature. Bull. Amer. Math. Soc. 84 (1978) 1239-1295.

[StW] Stein, E. M., Weiss, G., An Introduction to Fourier Analysis on Euclidean Spaces. Princeton University Press, 1971.

Recibido: 17 de enero de 1.997

Revisado: 18 de agosto de 1.997

Anthony Carbery ${ }^{* \dagger}$

Department of Mathematics

and Statistics

University of Edinburgh

James Clerk Maxwell Building

King's Buildings

Edinburgh EH9 3JZ, UNITED KINGDOM

carbery@maths.ed.ac.uk
Fulvio Ricci*

Dipartimento di Matematica

Politecnico di Torino

Corso Duca degli Abruzzi 24

10129 Torino, ITALY

fricci@polito.it

and

James Wright ${ }^{\dagger}$

Department of Mathematics

University of New South Wales

Sydney 2052

New South Wales, AUSTRALIA

jimw@math . unsw . edu . au

\footnotetext{
* Supported in part by EU HCM Fourier Analysis Programme ERB CHRX CT 93 0083

$\dagger$ Supported in part by EPSRC grants GR/H09713 and GR/L16576. The third author is also supported in part by an ARC grant
} 